\title{
Evaluation of postoperative analgesia in pediatric patients after hip surgery: lumbar plexus versus caudal epidural analgesia
}

This article was published in the following Dove Press journal: Journal of Pain Research

\author{
Mauricio Arce Villalobos' \\ Giorgio Veneziano ${ }^{1,2}$ \\ Rebecca Miller' \\ Ralph J Beltran ${ }^{1,2}$ \\ Senthil Krishna ${ }^{1,2}$ \\ Dmitry Tumin ${ }^{1,3}$ \\ Kevin Klingele ${ }^{1,2}$ \\ Joseph D Tobias ${ }^{1-3}$ \\ 'Department of Anesthesiology \& Pain \\ Medicine, Nationwide Children's \\ Hospital, Columbus, OH, USA; \\ ${ }^{2}$ Department of Anesthesiology \& Pain \\ Medicine, The Ohio State University \\ College of Medicine, Columbus, $\mathrm{OH}$, \\ USA; ${ }^{3}$ Department of Pediatrics, The \\ Ohio State University College of \\ Medicine, Columbus, $\mathrm{OH}$, USA
}

Background:There continues to be focus on the value of regional and neuraxial anesthetic techniques when combined with general anesthesia to improve postoperative analgesia. The reported advantages include decreased postoperative opioid requirements, decreased medication-related adverse effects, decreased hospital length of stay, and increased patient satisfaction. Orthopedic procedures of the hip may be amenable to such techniques as there is significant postoperative pain with the requirement for hospital admission and the administration of parenteral opioids. Given the surgical site, various regional anesthetic techniques may be used to provide analgesia including caudal epidural anesthesia (CEA) or lumbar plexus blockade (LPB).

Purpose: The objective of this study was to assess the effectiveness of LPB versus CEA as an analgesic thechnique for patients undergoing elective hip surgery from the opioid consumption and pain scores perspective.

Patients and methods: The current study retrospectively reviews our experience with CEA and LPB for postoperative analgesia after hip surgery in the pediatric population. Regional anesthesia technique was reviewed as well as opioid requirements and pain scores.

Results: The study cohort included 61 patients, 29 who received an LPB and 32 who received CEA. No difference was noted in the demographics between the two groups. Intraoperative opioid use was 0.7 (IQR: $0.5,1.1) \mathrm{mg} / \mathrm{kg}$ of oral morphine equivalents (MEs) in the LPB group compared to 0.6 (IQR: $0.5,0.9$ ) in the CEA group ( $p=0.479$ ). Postoperative opioid use over the first $48 \mathrm{hrs}$ was 4 (IQR: 1,6$) \mathrm{mg} / \mathrm{kg}$ of oral ME in the LPB group, compared to 2 (interquartile range [IQR]: 1 , 3 ) in the CEA group ( $p=0.103)$. Over the first $24 \mathrm{hrs}$ after surgery, the median pain score in the LPB group was 5 (IQR: $1-6)$, compared to 3 (IQR: 0,5$)$ in the CEA group $(p=0.014)$.

Conclusion: These retrospective data suggest a modest postoperative benefit of CEA when compared to LPB following hip surgery in the pediatric population. Postoperative pain scores were lower in patients receiving CEA; however, no difference in the intraoperative or postoperative opioid requirements was noted between the two groups.

Keywords: lumbar plexus block, caudal epidural anesthesia

\section{Introduction}

The use of regional anesthesia in the pediatric population has increased over the last decade as it offers several potential advantages in the provision of postoperative analgesia including a reduction in parenteral opioids, decreased exposure to general anesthetic agents, and shortened hospital length of stay. ${ }^{1,2}$ Such techniques may be particularly valuable following painful orthopedic procedures including hip and
Department of Anesthesiology \& Pain Medicine, Nationwide Children's Hospital, 700 Children's Drive, Columbus, Ohio 43205, USA

$\mathrm{Tel}+16147224200$

Fax +16147224203

Email Mauricio.

ArceVillalobos@nationwidechildrens.org 
femur surgery. ${ }^{3,4}$ Following such procedures, various regional anesthesia techniques may be used to provide analgesia including the lumbar plexus block (LPB) and caudal epidural anesthesia (CEA). ${ }^{5}$ LPB provides unilateral analgesia to the entire nerve distribution (femoral, lateral femoral cutaneous and obturator nerves) that provides sensation to the hip and avoids the need to enter the neuraxial space. ${ }^{6}$ Although CEA may be an easier technique, it provides bilateral analgesia in the distribution of the sacral and lower lumbar nerve roots. To better define the optimal analgesic technique, we retrospectively compared postoperative analgesia using either LPB or CEA in pediatric patients undergoing elective hip surgery.

\section{Methods}

The study was approved by the Institutional Review Board at Nationwide Children's Hospital (Columbus, Ohio), study ID IRB17-00862 and the need for informed consent was waived. We retrospectively analyzed the electronic medical records of patients between the ages of 10 and 17 years who had undergone elective hip surgery under general anesthesia with either a single-shot LPB or CEA over a 3-year period. All LPB blocks were performed under ultrasound guidance combined with nerve stimulator technology, while the CEA used only anatomical landmarks. The primary outcome was postoperative opioid consumption over the first 48 postoperative hours. Opioid administration was converted to oral morphine equivalents (MEs) for comparison. Secondary outcomes included intraoperative opioid requirements and median pain score (visual analog scale [VAS] or face, legs, activity, and cry consolability scale [FLACC]) depending on age and cognitive abilities. Pain scores were collected in the postanesthesia care unit (PACU) and at 2-hr intervals during the first $24 \mathrm{hrs}$ postoperatively.

Data were compared between groups using rank-sum tests, Chi-square tests, and Fisher's exact tests, as appropriate. Data were summarized as means with SD for continuous data, medians with interquartile range (IQR) for non-normally distributed data, and counts with percentages for categorical data. Normality of distribution was assessed using the Shapiro-Wilk tests. Data analysis was performed in Stata/IC 14.2 (College Station, StataCorp, LP). Two-tailed $P<0.05$ was considered statistically significant.

\section{Results}

The study cohort included 61 patients, 34 male and 27 female, with a median age of 14 years (IQR: 12, 16). Demographic data and the surgical procedures are outlined in Table 1. A LPB was placed in 29 patients (47\%) and a CEA was placed in 32 patients (53\%). In all cases, the CEA or LPB was supervised by an attending pediatric anesthesiologist with additional expertise in regional anesthesia. The LPB was performed by a pediatric anesthesiology fellow in $82 \%$ of the patients and by an attending pediatric anesthesiologist in $18 \%$. The CEA was performed in $47 \%$ of the patients by a pediatric anesthesiology fellow and in $53 \%$ of patients by

Table I Demographic data of the study cohorts

\begin{tabular}{|c|c|c|c|}
\hline \multirow[t]{3}{*}{ Characteristics } & \multicolumn{2}{|l|}{ Type of regional block } & \multirow[t]{3}{*}{$p$-value } \\
\hline & Lumbar plexus block $(\mathrm{N}=29)$ & Caudal block $(\mathrm{N}=32)$ & \\
\hline & Median (IQR), Mean \pm SD, or N (\%) & Median (IQR), Mean \pm SD, or N (\%) & \\
\hline Age (years) & $15(14,17)$ & $13(11,15)$ & 0.094 \\
\hline Female & $16(55 \%)$ & II (34\%) & 0.102 \\
\hline Height $(\mathrm{cm})$ & $158 \pm 13$ & $154 \pm 14$ & 0.247 \\
\hline Weight (kg) & $58 \pm 14$ & $54 \pm 24$ & 0.426 \\
\hline Body mass index $\left(\mathrm{kg} / \mathrm{m}^{2}\right)$ & $23 \pm 4$ & $23 \pm 7$ & 0.633 \\
\hline \multicolumn{4}{|l|}{ ASA status } \\
\hline 1 & $9(3 \mid \%)$ & $9(28 \%)$ & 0.858 \\
\hline 2 & $18(62 \%)$ & $19(59 \%)$ & \\
\hline 3 & $2(7 \%)$ & $4(13 \%)$ & \\
\hline Procedure types & $\begin{array}{l}\text { Periacetabular osteotomy, proximal femoral } \\
\text { osteotomy, surgical hip dislocation with osteot- } \\
\text { omy, labral repair }\end{array}$ & $\begin{array}{l}\text { Periacetabular osteotomy, proximal femoral } \\
\text { osteotomy, surgical hip dislocation with osteot- } \\
\text { omy, labral repair }\end{array}$ & \\
\hline
\end{tabular}

Abbreviations: ASA, American Society of Anesthesiologists; IQR, interquartile range; N, number. 
Table 2 Outcomes in pediatric patients age 10-17 years undergoing elective hip surgery according to type of regional block ( $\mathrm{N}=6 \mathrm{I})$

\begin{tabular}{|c|c|c|c|}
\hline \multirow[t]{3}{*}{ Outcomes } & \multicolumn{2}{|l|}{ Type of regional block } & \multirow[t]{3}{*}{ p-value } \\
\hline & Lumbar plexus block ( $\mathbf{N = 2 9 )}$ & Caudal block $(\mathrm{N}=32)$ & \\
\hline & Median (IQR), Mean \pm SD, or $\mathbf{N}(\%)$ & Median (IQR), Mean \pm SD, or $\mathbf{N}(\%)$ & \\
\hline Intraoperative opioids (ME/kg) & $0.7(0.5,1.1)$ & $0.6(0.5,0.9)$ & 0.479 \\
\hline Postoperative opioids (ME/kg) & $4.4(1.3,6.3)$ & $2.1(1.2,3.4)$ & 0.103 \\
\hline Median pain score & $5(1,6)$ & $3(0,5)$ & 0.014 \\
\hline Length of stay (days) & $3(2,3)$ & $2(2,3)$ & 0.303 \\
\hline
\end{tabular}

Abbreviations: ME, morphine equivalents; IQR, interquartile range; $\mathrm{N}$, number.

an attending pediatric anesthesiologist. The median age was 15 years in the LPB group and 13 years in the CEA group. For LPB, a solution consisting of ropivacaine $0.5 \%$ with $1: 200,000$ epinephrine and dexamethasone $(0.2 \mathrm{mg} / \mathrm{mL})$ was used at a median dose of $0.43 \mathrm{~mL} / \mathrm{kg}$ (IQR: $0.39,0.48$ ). CEA was accomplished with a solution of ropivacaine $0.2 \%$ with 1:200,000 epinephrine and clonidine $(1 \mu \mathrm{g} / \mathrm{kg})$ at a median dose of $0.46 \mathrm{~mL} / \mathrm{kg}$ (IQR: $0.38,0.64$ ). All patients in both study groups received acetaminophen (intravenous or oral in a dose of $10 \mathrm{mg} / \mathrm{kg}$ every $4 \mathrm{hrs}$ ) during the postoperative period as a part of the analgesic regimen. There was no significant difference in postoperative opioid consumption in the first $48 \mathrm{hrs}$ postoperatively between the LPB group and the CEA group. Postoperative opioid use over the first 48 hrs

was

$4.3 \mathrm{mg} / \mathrm{kg}$ (IQR: $1.3,6.3$ ) of oral ME in the LPB group, compared to $2.1 \mathrm{mg} / \mathrm{kg}$ (QR: 1.2, 3.4) in the CEA group (95\% CI of difference: $-2.9,0.3 ; p=0.103$ ) (Table 2). Over the first $24 \mathrm{hrs}$ after surgery, the median pain score in the LPB group was 5 (IQR: $1,-6$ ), compared to 3 (IQR: 0, 5) in the CEA group (95\% CI of difference: $-4,0 ; p=0.014)$. Other study outcomes, including intraoperative opioid use and length of stay, did not vary significantly according to the type of regional block received. Intraoperative opioid use was 0.7 (IQR: $0.5,1.1) \mathrm{mg} / \mathrm{kg}$ of oral ME in the LPB group compared to 0.6 (IQR: $0.5,0.9)$ in the CEA group (95\% CI of difference: $-0.2,0.1 ; p=0.479)$. Length of stay was 3 days (IQR: 2, 3) in the LPB group and 2 days (IQR: 2, 3) in the CEA group ( $95 \% \mathrm{CI}$ of difference: $-1,0 ; p=0.303$ ).

\section{Discussion}

Pain following orthopedic procedures can be severe and significantly impact the postoperative course of patients of all ages. Despite advances in functional outcomes with hip arthroplasty, providing excellent postoperative pain control remains challenging. Pain is a significant variable influencing recovery after hip arthroplasty. Inadequate pain control can lead to delayed mobilization, inability to engage in physical therapy, and increased length of hospital stay. ${ }^{2,6}$ Regional anesthesia has been shown to provide a wide spectrum of benefits such as early recovery, improved functional status, decreased postoperative nausea and vomiting, and decreased length of hospital stay. ${ }^{2,6}$

Given the location of the surgical procedure, various regional anesthetic techniques may be used to provide analgesia for procedures involving the hip and femur. Caudal anesthesia is one of the most popular regional blocks in children and is efficacious for postoperative analgesia for infraumbilical procedures. ${ }^{8}$ Although performed most commonly in infants and young children, CEA has been reported in adolescents and even adults as a means of providing postoperative analgesia. ${ }^{5,7,9,10}$ Anatomical changes that occur with aging may increase the difficulty of performing this technique in older children and adolescents. The development of a sacral fat pad may obscure bony landmarks and calcification of the sacrococcygeal ligament may make needle placement more challenging. Although not routinely used in our cohort of patients, ultrasound guidance may increase the success rate of CEA in adults. ${ }^{11}$ One of the major limitations of single-shot CEA is the relatively short duration of postoperative analgesia. As was used in our study population, adjuncts to the local anesthetic agents including clonidine may be used to prolong the duration of analgesia. ${ }^{12}$

While CEA remains a viable option for postoperative analgesia in adolescents following hip surgery, there remain limitations and concerns regarding the potential for adverse effects. CEA results in bilateral sensory and motor blockade which may limit ambulation immediately after surgery and may postpone physical therapy. Urinary retention is another common adverse effect with CEA and may result in the need for catheterization and its potential adverse effects. 
Sympathetic blockade and its associated hemodynamic effects including hypotension after CEA may be undesirable depending on patient comorbid conditions. ${ }^{9}$ Our current study was not designed to evaluate the incidence of adverse effects including urinary retention, hypotension, or the need for intraoperative intervention of hemodynamic effects between the two study groups.

Lumbar plexus block has gained more popularity in the pediatric population due to its potential advantages over neuraxial techniques, including a more prolonged duration of analgesia after single-shot techniques, unilateral blockade, and a decreased incidence of urinary retention. ${ }^{5,13}$ However, studies comparing LPB to neuraxial techniques for postoperative analgesia after major hip surgery have reported varying results. ${ }^{3,14}$ Additionally, despite the potential benefits of peripheral nerve blockade over neuraxial techniques, the LPB requires increased expertise and more time to perform. ${ }^{14,15}$

In our patient population, we noted no statistically significant difference in the primary outcome of opioid requirements in the first $48 \mathrm{hrs}$. Patients receiving CEA required less than half opioids in comparison to patients receiving a LPB (2.1 mg/kg versus $4.4 \mathrm{mg} / \mathrm{kg})$. As opioid-related adverse effects are generally dose related, this decrease in opioid requirements may translate into a clinical benefit. Analysis of postoperative pain scores revealed that CEA patients experienced less postoperative discomfort (median pain score of 3) compared to the LPB group (median pain score of 5); a finding that showed a statistical signifance in our study.

Given its retrospective nature, there are several inherent limitations of the current study including the inherent difficulties with grading analgesia through a retrospective chart review and the use of opioid requirements. Even though our retrospective study covered a 3-year period, only 61 patients fulfilled the study requirements, thereby limiting the power of the study. The solutions used to perform the blocks differed in the concentration of the local anesthetic agent and the adjunctive agent (clonidine for CEA and dexamethasone in LPB). The difference in the concentration of the local anesthetic agent is based on the need for a larger volume of solution for the CEA when compared to LPB and hence the need to limit the concentration to keep the total dose in accordance with local anesthetic dosing guidelines. The difference in the adjunctive agent was based on the routine
Table 3 Surgical procedures performed in pediatric patients age 10-17 years undergoing hip surgery according to type of regional block

\begin{tabular}{|l|l|l|}
\hline \multirow{2}{*}{ Surgical procedure } & \multicolumn{2}{|l|}{ Type of regional block } \\
\cline { 2 - 3 } & $\begin{array}{l}\text { Lumbar plexus } \\
\text { block (N=29) }\end{array}$ & $\begin{array}{l}\text { Caudal } \\
\text { block } \\
\mathbf{( N = 3 2 )}\end{array}$ \\
\hline Periacetabular osteotomy & $14(48 \%)$ & $6(18.75 \%)$ \\
Proximal femoral osteotomy & $2(6.8 \%)$ & $9(28 \%)$ \\
Surgical hip dislocation & $9(31 \%)$ & $12(37.5 \%)$ \\
Labral repair & $3(10.3 \%)$ & $3(9.3 \%)$ \\
Other & $1(3.4 \%)$ & $2(6.3 \%)$ \\
\hline
\end{tabular}

clinical practice at our hospital. Furthermore, although the specific types of surgical procedures were constant among the cohorts, there was some variation in the numbers of the specific procedures between the two groups (Table 3). Given the variation in the surgical procedure, there may be some variation in the innervation of the surgical site and hence the nerve distribution (lumbar \pm sacral plexus) required to provide analgesia. The regional anesthesia techniques used in this study differ in that aspect as the LPB does not reliably provide coverage of the sciatic nerve and sacral plexus, while CEA covers both lumbar and sacral dermatomes including the lumbar plexus and the sacral plexus. Furthermore, as already noted, given the retrospective nature of our study, a true evaluation of adverse effects was not feasible. There was also a difference in the experience level of those directly providing the regional anesthetic. One final limitation of the study was that a safety profile specifically evaluating adverse effects of the two techniques was not included as part of the study protocol and outcome analysis.

With these limitations in mind, our retrospective analysis of adolescents undergoing hip surgery provides additional information regarding the optimal regional anesthetic technique for providing analgesia following hip surgery. Postoperative pain scores were lower in patients who received CEA. Although no statistical significance was noted in total postoperative opioid requirements, there was a greater than $50 \%$ decrease in total needs in patients who received CEA.

\section{Disclosure}

The authors report no conflicts of interest in this work. 


\section{References}

1. Schloss B, Bhalla T, Klingele K, Phillips D, Prestwich B, Tobias JD. A retrospective review of femoral nerve block for postoperative analgesia after knee surgery in the pediatric population. J Pediatr Orthop. 2014;34(4):459-461. doi:10.1097/ BPO.0000000000000113

2. Capdevila X, Barthelet Y, Biboulet P, Ryckwaert Y, Rubenovitch J, d 'Athis F. Effects of perioperative analgesic technique on the surgical outcome and duration of rehabilitation after major knee surgery. Anesthesiology. 1999;91(1):8-15.

3. Omar AM, Mansour MA, Kamal AS. Psoas compartment block for acute postoperative pain management after hip surgery in pediatrics, a comparative study with caudal analgesia. Reg Anesth Pain Med. 2011;36(2):121-124. doi:10.1097/AAP.0b013e31820d41f3

4. Boretsky K, Hernandez MA, Eastburn E, Sullivan C. Ultrasoundguided lumbar plexus block in children and adolescents using a transverse lumbar paravertebral sonogram: initial experience. Paediatr Anaesth. 2018;28(3):291-295. doi:10.1111/pan.13328

5. Schloss B, Martin D, Tripi J, Klingele K, Tobias JD. Caudal epidural blockade for major orthopedic hip surgery in adolescents. Saudi J Anaesth. 2015;9(2):128-131. doi:10.4103/1658-354X.152832

6. Touray ST, de Leeuw MA, Zuurmond WW, Perez RS. Psoas compartment block for lower extremity surgery: a meta-analysis. Br J Anaesth. 2008;101(6):750-760. doi:10.1093/bja/aen298

7. Manion SC, Tobias JD. Lumbar plexus blockade in children. Amer J Pain Manage. 2005;15:120-126.
8. Jöhr M, Berger TM. Caudal blocks. Paediatr Anaesth. 2012;22 (1):44-50. doi:10.1111/j.1460-9592.2011.03669.x

9. Kiribayashi M, Inagaki Y, Nishimura Y, Yamasaki K, Takahashi S, Ueda K. Caudal blockade shortens the time to walking exercise in elderly patients following low back surgery. J Anesth. 2010;24 (2):192-196. doi:10.1007/s00540-009-0840-6

10. Kita T, Maki N, Song YS, Arai F, Nakai T. Caudal epidural anesthesia administered intraoperatively provides for effective postoperative analgesia after total hip arthroplasty. J Clin Anesth. 2007;19 (3):204-208. doi:10.1016/j.jclinane.2006.10.011

11. Chen CP, Tang SF, Hsu TC, et al. Ultrasound guidance in caudal epidural needle placement. Anesthesiology. 2004;101(1):181-184.

12. Lee JJ, Rubin AP. Comparison of a bupivacaine-clonidine mixture with plain bupivacaine for caudal analgesia in children. Br J Anaesth. 1994;72(3):258-262.

13. Singelyn FJ, Gouverneur JMA. Postoperative analgesia after total hip arthroplasty: IV PCA with morphine, patient controlled epidural analgesia, or continuous "3-in-1" block?: a prospective evaluation by our acute pain service in more than 1300 patients. J Clin Anesth. 1999;11(7):550-554.

14. Dadure C, Bringuier S, Mathieu O, et al. Continuous epidural block versus continuous psoas compartment block for postoperative analgesia after major hip or femoral surgery in children: a prospective comparative randomized study. Ann Fr Anesth Reanim. 2010;29 (9):610-615. doi:10.1016/j.annfar.2010.05.033

15. Walker BJ, Flack SH, Bosenberg AT. Predicting lumbar plexus depth in children and adolescents. Anesth Analg. 2011;112(3):661-665. doi:10.1213/ANE.0b013e318207c538

\section{Publish your work in this journal}

The Journal of Pain Research is an international, peer reviewed, open access, online journal that welcomes laboratory and clinical findings in the fields of pain research and the prevention and management of pain. Original research, reviews, symposium reports, hypothesis formation and commentaries are all considered for publication. The manuscript management system is completely online and includes a very quick and fair peer-review system, which is all easy to use. Visit http:// www.dovepress.com/testimonials.php to read real quotes from published authors. 